\title{
Decompositions of the Boolean lattice into rank-symmetric chains
}

\author{
István Tomon \\ Department of Pure Mathematics and Mathematical Statistics \\ University of Cambridge \\ Cambridge, United Kingdom. \\ it262@cam.ac.uk
}

Submitted: Jun 13, 2015; Accepted: Jun 12, 2016; Published: Jun 24, 2016

Mathematics Subject Classifications: 05A16, 06A07

\begin{abstract}
The Boolean lattice $2^{[n]}$ is the power set of $[n]$ ordered by inclusion. A chain $c_{0} \subset \cdots \subset c_{k}$ in $2^{[n]}$ is rank-symmetric, if $\left|c_{i}\right|+\left|c_{k-i}\right|=n$ for $i=0, \ldots, k$; and it is symmetric, if $\left|c_{i}\right|=(n-k) / 2+i$. We prove that there exist a bijection

$$
p:[n]^{(\geqslant n / 2)} \rightarrow[n]^{(\leqslant n / 2)}
$$

and a partial ordering $<$ on $[n]^{(\geqslant n / 2)}$ satisfying the following properties:

- $\subset$ is an extension of $<$ on $[n]^{(\geqslant n / 2)}$;

- if $C \subset[n]^{(\geqslant n / 2)}$ is a chain with respect to $<$, then $p(C) \cup C$ is a rank-symmetric chain in $2^{[n]}$, where $p(C)=\{p(x): x \in C\}$;

- the poset $\left([n]^{(\geqslant n / 2)},<\right)$ has the so called normalized matching property.

We show two applications of this result.

A conjecture of Füredi asks if $2^{[n]}$ can be partitioned into $\left(\begin{array}{c}n \\ \lfloor n / 2\rfloor\end{array}\right)$ chains such that the size of any two chains differ by at most 1 . We prove an asymptotic version of this conjecture with the additional condition that every chain in the partition is rank-symmetric: $2^{[n]}$ can be partitioned into $\left(\begin{array}{c}n \\ \lfloor n / 2\rfloor\end{array}\right)$ rank-symmetric chains, each of size $\Theta(\sqrt{n})$.

Our second application gives a lower bound for the number of symmetric chain partitions of $2^{[n]}$. We show that $2^{[n]}$ has at least $2^{\Omega\left(2^{n} \log n / \sqrt{n}\right)}$ symmetric chain partitions.
\end{abstract}

Keywords: chain decompositions; Boolean lattice 


\section{Introduction}

Let us introduce the main definitions and notation used throughout the paper. The notation is mostly standard and can be found in [1], for example.

The Boolean lattice $2^{[n]}$ is the power set of $[n]=\{1, \ldots, n\}$ ordered by inclusion. The family of $k$ element subsets of $[n]$ is denoted by $[n]^{(k)}$; also $[n]^{(\leqslant k)}=\left\{x \in 2^{[n]}:|x| \leqslant k\right\}$, and define $[n]^{(\geqslant k)}$ similarly.

A chain in a poset is a subset of pairwise comparable elements. A chain $C \subset 2^{[n]}$ with elements $c_{0} \subset \cdots \subset c_{k}$ is rank-symmetric, if $\left|c_{i}\right|+\left|c_{k-i}\right|=n$, and $C$ is skipless, if $\left|c_{i}\right|=\left|c_{0}\right|+i$ for $i=0, \ldots, k$. A chain is symmetric, if it is rank-symmetric and skipless.

A poset $P$ is graded if there exists a partition of its elements into subsets $A_{0}, A_{1}, \ldots, A_{n}$ such that $A_{0}$ is the set of minimal elements, and whenever $x \in A_{i}$ and $x<y$ with no $x<z<y$, then $y \in A_{i+1}$. If there exists such a partition, then it is unique and $A_{0}, A_{1}, \ldots, A_{n}$ are the levels of $P$. If $x \in A_{i}$, then the rank of $x$ is $i$ and it is denoted by $r k(x)$.

A graded poset $P$ is unimodal if there exists $0 \leqslant m \leqslant n$ such that $\left|A_{0}\right| \leqslant \ldots \leqslant\left|A_{m}\right|$ and $\left|A_{m}\right| \geqslant\left|A_{m+1}\right| \geqslant \ldots \geqslant\left|A_{n}\right|$. Also, $P$ is rank-symmetric, if we have $\left|A_{i}\right|=\left|A_{n-i}\right|$ for $i=0, \ldots, n$.

A bipartite graph $G=(A, B, E)$ is a normalized matching graph, if for any $X \subset A$ we have

$$
\frac{|X|}{|A|} \leqslant \frac{|\Gamma(X)|}{|B|}
$$

where $\Gamma(X)$ is the set of neighbours of $A$ in $B$. A graded poset $P$ is a normalized matching poset (or satisfies the normalized matching property), if the bipartite comparability graph induced on any two levels $A_{i}$ and $A_{j}$ is a normalized matching graph for $i, j \in\{0, \ldots, n\}$, $i \neq j$.

It is easy to show that $2^{[n]}$ is a rank-symmetric, unimodal normalized matching poset.

By the classical theorem of Sperner [15], the minimum number of chains $2^{[n]}$ can be partitioned into is $\left(\begin{array}{c}n \\ \lfloor n / 2\rfloor\end{array}\right)$. Also, Brujin et al. [2] showed that $2^{[n]}$ admits a chain decomposition into $\left(\begin{array}{c}n \\ \lfloor n / 2\rfloor\end{array}\right)$ symmetric chains; later Griggs [7] extended their result by proving that every rank-symmetric, unimodal normalized matching poset has a symmetric chain decomposition.

The aim of this paper is to show the existence of a relatively "rich" subposet of $\left(2^{[n]}, \subset\right)$, where every chain corresponds to a rank-symmetric chain in $2^{[n]}$, and every chain partition corresponds to a chain partition of $2^{[n]}$ into rank-symmetric chains. Here, rich means that the poset has the normalized matching property. Our main result is the following.

Theorem 1. For every positive integer $n$, there exist a bijection

$$
p:[n]^{(\geqslant n / 2)} \rightarrow[n]^{(\leqslant n / 2)}
$$

and a partial ordering $<$ on $[n]^{(\geqslant n / 2)}$ such that

(i) $\subset$ is an extension of $<$; 
(ii) if $C \subset[n]^{(\geqslant n / 2)}$ is a chain with respect to $<$, then $C \cup p(C)$ is a rank-symmetric chain in $2^{[n]}$, where $p(C)=\{p(x): x \in C\}$;

(iii) the poset $\left([n]^{(\geqslant n / 2)},<\right)$ satisfies the normalized matching property.

We also show two applications of this theorem.

The first application is the following rank-symmetric variant of a problem of Füredi. Note that in a symmetric chain decomposition of $2^{[n]}$, we must have $\left(\begin{array}{c}n \\ i\end{array}\right)-\left(\begin{array}{c}n \\ i-1\end{array}\right)$ chains of size $n+1-2 i$ for $i=0, \ldots,\lfloor n / 2\rfloor$. Vaguely saying, this chain decomposition contains short and long chains as well. In contrast to this observation, Füredi [5] proposed the following problem.

Conjecture 2. Let $n$ be a positive integer. The Boolean lattice $2^{[n]}$ can be partitioned into $\left(\begin{array}{c}n \\ \lfloor n / 2\rfloor\end{array}\right)$ chains such that the size of any two chains differ by at most 1 .

If the conjecture is true, it means that $2^{[n]}$ admits a chain partition into $\left(\begin{array}{c}n \\ \lfloor n / 2\rfloor\end{array}\right)$ chains such that the size of each chain is $\approx \sqrt{\pi / 2} \sqrt{n}$. While this conjecture is still open, there are a few partial results. Hsu, Logan, Shahriari and Towse [9] proved that there exists a chain partition into $\left(\begin{array}{c}n \\ \lfloor n / 2\rfloor\end{array}\right)$ skipless chains such that the size of each chain is at least $\sqrt{n} / 2+O(1)$. Also, the author of this paper [16] proved that there is a chain partition of $2^{[n]}$ into $\left(\begin{array}{c}n \\ \lfloor n / 2\rfloor\end{array}\right)$ chains such that the size of each chain is between $0.8 \sqrt{n}$ and $13 \sqrt{n}$. We also proposed the following rank-symmetric version of Conjecture 2 .

Conjecture 3. Let $n$ be a positive integer. The Boolean lattice $2^{[n]}$ can be partitioned into $\left(\begin{array}{c}n \\ \lfloor n / 2\rfloor\end{array}\right)$ rank-symmetric chains such that the size of any two chains differ in at most 2.

To demonstrate the difficulty of the problem, we challenge the reader to think about the following much weaker result: if $h$ is fixed and $n$ is sufficiently large, then $2^{[n]}$ can be partitioned into rank-symmetric chains, each of size at least $h$. While this problem is not too hard in the case we do not demand our chains to be rank-symmetric, we have to overcome extra obstacles in the rank-symmetric case.

In this paper, we prove the following result concerning Conjecture 3.

Theorem 4. Let

$$
\alpha=\sqrt{2} \sum_{k=2}^{\infty} \frac{\sqrt{\log k}-\sqrt{\log (k-1)}}{k} \approx 0.8482 .
$$

For any $\epsilon>0$ there exists an $N_{\epsilon}$ such that if $n>N_{\epsilon}$, then $2^{[n]}$ can be partitioned into $\left(\begin{array}{c}n \\ \lfloor n / 2\rfloor\end{array}\right)$ rank-symmetric chains, all of them of size between $(\alpha-\epsilon) \sqrt{n}$ and $O(\sqrt{n} / \epsilon)$.

In particular, if $n$ is sufficiently large, there is a chain partition of $2^{[n]}$ into $\left(\begin{array}{c}n \\ \lfloor n / 2\rfloor\end{array}\right)$ rank-symmetric chains such that the size of each chain is between $0.8 \sqrt{n}$ and $13 \sqrt{n}$.

We note that this is exactly the same result as Theorem 1.5 in [16], but with the additional condition that our chains are rank-symmetric. 
With our second application, we show that the poset $\left([n]^{(\geqslant n / 2)},<\right)$ can be used to construct a lot of different symmetric chain decompositions of $2^{[n]}$. We deduce the following lower bound on the number of symmetric chain partitions of $2^{[n]}$.

Theorem 5. The Boolean lattice $2^{[n]}$ has at least

$$
2^{\Omega\left(2^{n} \log n / \sqrt{n}\right)}
$$

different partitions into $\left(\begin{array}{c}n \\ \lfloor n / 2\rfloor\end{array}\right)$ symmetric chains.

In the proof, we also establish a nontrivial lower bound for the number of matchings in an arbitrary normalized matching graph.

\section{The proof of Theorem 1}

In this section, we prove Theorem 1. The proof relies on the following well known symmetric chain decomposition of $2^{[n]}$, which we shall refer to as the canonical symmetric chain decomposition (CSCD). The CSCD was first introduced by De Bruijn, Tengbergen, Kruyswijk [2] in a more general setting, and later by Kleitman [12]. Let us define this chain partition.

The most convenient way to define the CSCD is by induction. In case $n=1$, this chain decomposition is formed by one chain: $\{\emptyset,\{1\}\}$. Let $m=\left(\begin{array}{c}n-1 \\ \lfloor(n-1) / 2\rfloor\end{array}\right)$ and let $C_{1}, \ldots, C_{m}$ be the chains in the CSCD of $2^{[n-1]}$. The CSDC of $2^{[n]}$ is defined as follows. For $i=1, \ldots, m$, define the chains $C_{i}^{1}$ and $C_{i}^{2}$ such that if the elements of $C_{i}$ are $x_{1} \subset \cdots \subset x_{k}$, then

$$
\begin{gathered}
C_{i}^{1}=C_{i} \cup\left\{x_{k} \cup\{n\}\right\}, \\
C_{i}^{2}=\left\{x_{i} \cup\{n\}: 1 \leqslant i \leqslant k-1\right\} .
\end{gathered}
$$

One can easily check that the nonempty chains among $\left\{C_{i}^{j}\right\}_{i=1, \ldots, m}^{j=1,2}$ form a symmetric chain decomposition of $2^{[n]}$. We set this as the CSCD of $2^{[n]}$.

For simplicity, write $Q=[n]^{(\geqslant n / 2)}$. Now we shall define our bijection $p: Q \rightarrow[n]^{(\leqslant n / 2)}$ with respect to the CSCD. Let $x \in Q$ and let $C$ be the unique chain in the CSCD containing $x$. As $C$ is symmetric, $C$ contains a unique element $y$ such that $|y|+|x|=n$. Set $p(x)=y$.

Define the relation $<$ on $Q$ as follows. Let $y<x$ if

$$
p(x) \subset p(y) \subset y \subset x .
$$

As $\subset$ is a partial order, $<$ is also a partial order. Hence, $(Q,<)$ is a partially ordered set. Furthermore, $Q$ has the following important property: if $C$ is a chain in $(Q,<)$, then $C \cup p(C)$ is a rank-symmetric chain in $\left(2^{[n]}, \subset\right)$, where $p(C)=\{p(x): x \in C\}$. Hence, our task is reduced to proving the following theorem. 
Theorem 6. The graded poset $(Q,<)$ is a normalized matching poset.

To prove this theorem, we first analyze the properties of the CSCD of $2^{[n]}$. Instead of using the inductive construction, we shall rely on the following description of the CSCD, discovered by Greene and Kleitman [6], and independently Leeb (unpublished).

First, let us introduce some notation. If $v$ is an element of a cartesian product with $d$ terms and $i \in[d]$, then $v_{i}$ denotes the $i$-th coordinate of $v$. As usual, let $[i, j]=$ $\{i, i+1, \ldots, j\}$ for $i, j$ integers with $i<j$. Also, for $x \in 2^{[n]}$, let

$$
c_{x}(i, j)=|x \cap[i, j]|-|[i, j] \backslash x| .
$$

The signature of $x$, denoted by $s g(x)$, is an element of $\{0,1, *\}^{n}$ defined as follows.

If $i \in x$ and there exists an integer $j$ with $i<j \leqslant n$ such that $c_{x}(i, j)=0$, then $s g(x)_{i}=1$. The smallest such $j$ is the pair of $i$ in $x$ and is denoted by $\operatorname{pr}_{x}(i)$. If there is no such $j$, then $s g(x)_{i}=*$.

If $i \notin x$ and there exists an integer $j$ with $1 \leqslant j<i$ such that $c_{x}(j, i)=0$, then $s g(x)_{i}=0$. The largest such $j$ is the pair of $i$ in $x$ and is denoted by $p r_{x}(i)$. If there is no such $j$, then $s g(x)_{i}=*$.

Let $*(x)=\left\{i \in[n]: s g(x)_{i}=*\right\}$, and define $0(x)$ and $1(x)$ similarly. Also, let $I(x)=\left\{\left[i, p r_{x}(i)\right]: i \in 1(x)\right\}$ be the set of intervals whose endpoints are the pairs of $x$.

For example, if $x=\{2,3,4,6,7,10,11\} \in 2^{[12]}$, then

$$
s g(x)=(*, *, *, \overline{1,0}, \overline{1, \overline{1,0}, 0}, *, \overline{1,0}),
$$

so $*(x)=\{1,2,3,10\}, 1(x)=\{4,6,7,11\}$ and $0(x)=\{5,8,9,12\}$.

The signature of $x$ can be interpreted in another way as well. Using our earlier example, write $x$ as ()$)())(())($, where ")" represents the elements in $x$ and "(" represents the elements not in $x$. Then, we have $s g(x)_{i}=*$ if the bracket representing $i$ is invalid, in other words does not have a pair.

Define the equivalence relation $\sim$ on $2^{[n]}$ such that $x \sim y$ if $s g(x)=s g(y)$. The result of Greene and Kleitman [6] is the following connection between the signature and the CSCD.

Proposition 7. The complete equivalence classes of $\sim$ are the chains in the CSCD.

Now we shall list some of the most important properties of the signature. We avoid their proof, as they can be easily verified by the reader. Also, we shall present a proof of the properties listed in Proposition 10, which are quite similar to the ones we describe now.

Proposition 8. Let $x \in 2^{[n]}$.

(i) If $i \in[n]$ and $j=p r_{x}(i)$ exists, then $p r_{x}(j)$ exists as well and $p r_{x}(j)=i$. Also, $s g(x)_{i}=1-s g(x)_{j}$.

(ii) $\max (*(x) \backslash x)<\min (x \cap *(x))$. 
(iii) Any two intervals in $I(x)$ are either disjoint, or one is contained in the other. Also, the intervals are disjoint from $*(x)$.

In the light of Proposition 7 and Proposition 8, let us propose the following more useable description of $<$.

Proposition 9. Let $k \geqslant n / 2+1, x \in[n]^{(k)}$, and let $i_{1}<\cdots<i_{t}$ be the elements of $x \cap *(x)$. Also, let $b \in x$ and $y=x \backslash\{b\}$. Then $y<x$ if and only if $b=i_{u}$ with $u \in[2 k-n]$.

Proof. Let $C$ be the $\sim$ equivalence class of $x$. The elements of $C$ not larger than $x$ have the form $1(x) \cup\left\{i_{u+1}, \ldots, i_{t}\right\}$ with $u=0, \ldots, t$. As we have $|1(x)|=k-t$, the pair of $x$ is $p(x)=1(x) \cup\left\{i_{2 k-n+1}, \ldots, i_{t}\right\}$.

Suppose that $y<x$. As $p(x) \subset y$, we must have $b=i_{u}$ with some $u \in[2 k-n]$.

Now we only need to show that in this case, $p(x) \subset p(y)$ holds as well. If $b=i_{1}$, then $p(y), p(x), x, y$ are all elements of $C$, hence we are done.

Now suppose that $b=i_{u}$ with $u \in\{2, \ldots, 2 k-n\}$. Look at the interval $J=\left[i_{u-1}+\right.$ $\left.1, i_{u}-1\right]$. This interval is disjoint from $*(x)$, so it is the union of disjoint intervals $\left[a_{1}, b_{1}\right], \ldots,\left[a_{r}, b_{r}\right] \in I(x)$. Hence,

$$
c_{x}\left(i_{u-1}, i_{u}\right)=2+\sum_{j=1}^{r} c_{x}\left(a_{j}, b_{j}\right)=2 .
$$

But then $c_{y}\left(i_{u-1}, i_{u}\right)=0$, so $s g(y)_{i_{u-1}}=1$ and $s g(y)_{i_{u}}=0$. Also, $s g(x)$ and $s g(y)$ agrees on every coordinate other than $i_{u-1}$ and $i_{u}$. Thus,

$$
y=1(y) \cup\left\{i_{1}, \ldots, i_{t}\right\} \backslash\left\{i_{u}\right\}
$$

and

$$
p(y)=1(y) \cup\left\{i_{2 k-n+1}, \ldots, i_{t}\right\}=p(x) \cup\left\{i_{u-1}\right\} .
$$

This shows that $p(x) \subset p(y)$.

By Proposition 9, the bipartite comparability graph of $(Q,<)$ induced on the levels $[n]^{(k-1)}$ and $[n]^{(k)}$ is $2 k-n$ regular from $[n]^{(k)}$. However, it is not regular from $[n]^{(k-1)}$. Also, while this proposition lets us identify the neighbours of any $x \in[n]^{(k)}$ in $[n]^{(k-1)}$ easily, finding a description of the neighbours of $y \in[n]^{(k-1)}$ in $[n]^{(k)}$ is more troublesome.

However, we shall overcome this obstacle by slightly modifying the definition of the signature. We introduce the circular signature of an element $x \in 2^{[n]}$. The circular signature of $x$ is denoted by $\operatorname{csg}(x)$, and is an element of $\{0,1, *\}^{n}$ defined as follows.

View $x$ as a subset of $\mathbb{Z}_{n}$, the ring of integers modulo $n$. For $i, j \in \mathbb{Z}_{n}$, define the interval $[i, j]_{n} \subset \mathbb{Z}_{n}$ such that

$$
[i, j]_{n}= \begin{cases}{[i, j]} & \text { if } i \leqslant j \\ {[i, n] \cup[1, j]} & \text { if } i>j\end{cases}
$$




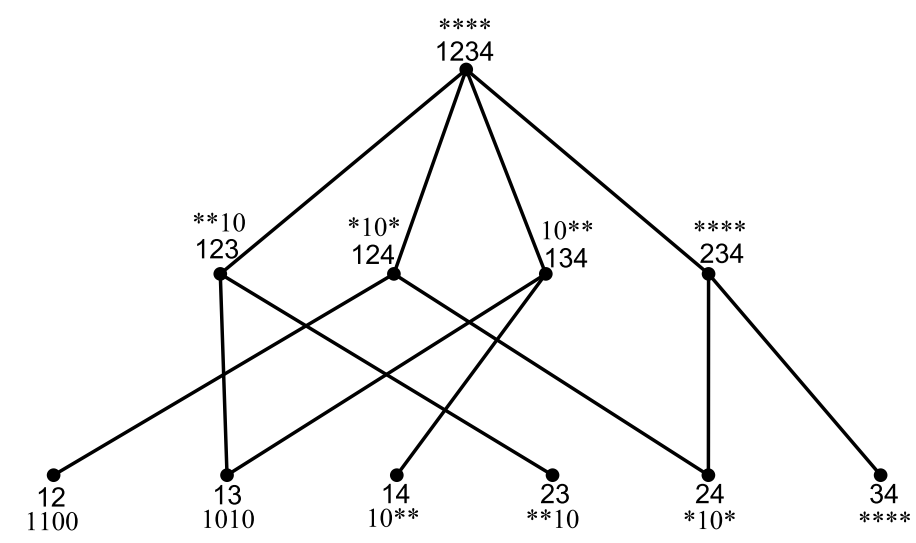

Figure 1: The poset $(Q,<)$ for $n=4$.

For $x \subset 2^{[n]}$, define the function

$$
c_{x}^{\prime}(i, j)=\left|[i, j]_{n} \cap x\right|-\left|[i, j]_{n} \backslash x\right| .
$$

If $i \in x$ and there exists $j \in \mathbb{Z}_{n}$ such that $c_{x}^{\prime}(i, j)=0$, then $\operatorname{csg}(x)_{i}=1$ and the circular pair of $i$ in $x$ is the $j$ with this property for which $\left|[i, j]_{n}\right|$ is the smallest and is denoted by $\operatorname{pr}_{x}^{\prime}(i)$. If there is no such $j$, then $\operatorname{csg}(x)_{i}=*$.

If $i \notin x$ and there exists $j \in \mathbb{Z}_{n}$ such that $c_{x}(j, i)=0$, then $\operatorname{csg}(x)_{i}=0$ and the circular pair of $i$ in $x$ is the $j$ with this property for which $\left|[j, i]_{n}\right|$ is the smallest and is denoted by $\operatorname{pr}_{x}^{\prime}(i)$. If there is no such $j$, then $\operatorname{csg}(x)_{i}=*$.

Also, let $*^{\prime}(x)=\left\{i \in[n]: \operatorname{csg}(x)_{i}=*\right\}$ and define $0^{\prime}(x)$ and $1^{\prime}(x)$ similarly. Finally, let $I^{\prime}(x)=\left\{\left[i, p r_{x}(i)\right]_{n}: i \in 1^{\prime}(x)\right\}$.

We note that the idea of circular signature can be also found in [11]. Taking our earlier example $x=\{2,3,4,6,7,10,11\} \in 2^{[12]}$, now we have

$$
\operatorname{csg}(x)=(\overline{0}, *, *, \overline{1,0}, \overline{1, \overline{1,0}, 0}, \overline{1, \overline{1,0}}) .
$$

Also, $*^{\prime}(x)=\{2,3\}, 0^{\prime}(x)=\{1,5,8,9,12\}$ and $1^{\prime}(x)=\{4,6,7,10,11\}$.

The following proposition lists the properties of the circular signature and helps us compare it with the signature.

Proposition 10. Let $x \in[n]^{(k)}$ with $n / 2 \leqslant k \leqslant n$. Let $i_{1}<\cdots<i_{t}$ be the elements of $*(x) \cap x$ and $j_{1}<\cdots<j_{t-2 k+n}$ be the elements of $*(x) \backslash x$.

(i) If $i \in[n]$ and $j=p r_{x}^{\prime}(i)$ exists, then $\operatorname{pr}_{x}^{\prime}(j)$ exists and $i=p r_{x}^{\prime}(j) . A l s o, \operatorname{csg}(x)_{i} \neq$ $\operatorname{csg}(x)_{j}$.

(ii) If $i \in[n] \backslash *(x)$, then $\operatorname{csg}(x)_{i}=\operatorname{sg}(x)_{i}$ and $p r_{x}(i)=p r_{x}^{\prime}(i)$. 
(iii) Any two intervals in $I^{\prime}(x)$ are either disjoint or one is contained in the other. Also, the intervals are disjoint from $*^{\prime}(x)$.

(iv) If $1 \leqslant r \leqslant 2 k-n$, then $\operatorname{csg}(x)_{i_{r}}=*$.

(v) If $2 k-n<r \leqslant t$, then $\operatorname{csg}(x)_{i_{r}}=1$ and $\operatorname{pr}_{x}^{\prime}\left(i_{r}\right)=j_{t+1-r}$. Also, if $1 \leqslant s \leqslant t-2 k+n$, then $\operatorname{csg}(x)_{j_{s}}=0$ and $\operatorname{pr}_{x}^{\prime}\left(j_{s}\right)=i_{t+1-s}$.

Proof. (i) Look at case $i \in x$, the proof in the other case is similar. The function $c_{x}^{\prime}(i, y)$ changes by 1 as $y$ changes by $1 \bmod n$, and $c_{x}^{\prime}(i, i)=1$. Hence, $c_{x}^{\prime}(i, y)>0$ for all $y \in[i, j-1]_{n}$, and so we must have $j \notin x$, proving that $\operatorname{csg}(x)_{i}=1-\operatorname{csg}(x)_{j}$.

Also, we have $c_{x}^{\prime}(i, j)=0$, so $p r_{x}^{\prime}(j)$ exists. Let $i^{\prime} \in[n]$ be such that $c_{x}^{\prime}\left(i^{\prime}, j\right)=0$ and $\left|\left[i^{\prime}, j\right]_{n}\right|$ is minimal. Then $i^{\prime} \in[i, j]$. If $i \neq i^{\prime}$, then $c_{x}^{\prime}\left(i, i^{\prime}\right)=c_{x}^{\prime}(i, j)-c_{x}^{\prime}\left(i^{\prime}, j\right)=0$ and $\left|\left[i, i^{\prime}\right]_{n}\right|<\left|[i, j]_{n}\right|$, which is a contradiction.

(ii) If $i<j$, then $c_{x}(i, j)=c_{x}^{\prime}(i, j)$, so this part trivially follows.

(iii) Let $[a, b]_{n} \in I^{\prime}(x)$. The function $c_{x}^{\prime}(a, y)$ changes by 1 as $y$ changes by $1 \bmod n$. Also, $c_{x}^{\prime}(a, a)=1$, so $c_{x}^{\prime}(a, y)>0$ for all $y \in[a, b]_{n}, y \neq b$. Let $c \in[a, b]_{n}, c \neq a, b$. Then $c_{x}^{\prime}(c, b)<0$. But $c_{x}^{\prime}(c, c)=1$, hence there exist $d \in[c, b-1]_{n}$ such that $c_{x}^{\prime}(c, d)=0$. This means that $[a, b]_{n}$ is disjoint from $*^{\prime}(x)$, and every interval in $I^{\prime}(x)$ intersecting $[a, b]_{n}$ is either contained in $[a, b]_{n}$ or contains $[a, b]_{n}$.

(iv)-(v) If $c_{x}^{\prime}\left(i_{r}, j\right)=0$ for some $j \in[n]$, then the $j$ for which $\left|\left[i_{r}, j\right]_{n}\right|$ is minimal, must be equal to one of $j_{1}, \ldots, j_{t-2 k+n}$. Suppose $j=j_{r^{\prime}}$. As $j_{r^{\prime}}<i_{r}$, we have $c_{x}^{\prime}\left(i_{r}, j_{r^{\prime}}\right)=$ $c_{x}\left(i_{r}, n\right)+c_{x}\left(1, j_{r^{\prime}}\right)$. Let $\left[a_{1}, b_{1}\right], \ldots,\left[a_{s}, b_{s}\right]$ be the maximal intervals in $I(x)$ contained in $\left[i_{r}, n\right]$. Then,

$$
c_{x}\left(i_{r}, n\right)=t-r+1+\sum_{l=1}^{s} c_{x}\left(a_{l}, b_{l}\right)=t-r+1 .
$$

We can show similarly that $c_{x}\left(1, j_{r^{\prime}}\right)=-r^{\prime}$. Hence, $c_{x}^{\prime}\left(i_{r}, j_{r^{\prime}}\right)=0$ if and only if $r^{\prime}=$ $t-r+1$.

Now we can describe the neighbours of any element with the help of the circular signature.

Proposition 11. (i) Let $x \in[n]^{(k)}$ with $n / 2 \leqslant k \leqslant n$ and let $i_{1} \cdots<i_{2 k-n}$ be the elements of $*^{\prime}(x)$. The elements in $[n]^{(k-1)}$ that are $<-$ smaller than $x$ are $x \backslash$ $\left\{i_{1}\right\}, \ldots, x \backslash\left\{i_{2 k-n}\right\}$. Furthermore, for $u=1, \ldots, 2 k-n$, the interval $\left[i_{u-1}, i_{u}\right]_{n}$ is a maximal interval in $I^{\prime}\left(x \backslash\left\{i_{u}\right\}\right)$, where the index $u-1$ meant modulo $2 k-n$.

(ii) Also, if $y \in[n]^{(k-1)}$ and $\left[j_{1}, p r_{y}\left(j_{1}\right)\right]_{n}, \ldots,\left[j_{s}, p r_{y}\left(j_{s}\right)\right]_{n}$ are the maximal intervals in $I^{\prime}(y)$, then there are s elements in $[n]^{(k)}$ that are $<$-larger than $y$, namely $y \cup p r_{y}^{\prime}\left(j_{r}\right)$ for $r=1, \ldots, s$.

Proof. (i) The first part of (i) is straightforward from Proposition 9 and Proposition 10. Now let $z=x \backslash\left\{i_{u}\right\}$. We have

$$
c_{z}^{\prime}(i, j)= \begin{cases}c_{x}^{\prime}(i, j) & \text { if } i_{u} \notin[i, j]_{n} \\ c_{x}^{\prime}(i, j)-2 & \text { if } i_{u} \in[i, j]_{n} .\end{cases}
$$


If $i \in 1^{\prime}(x)$, then $\left[i, p r_{x}^{\prime}(i)\right]_{n}$ does not contain $i_{u}$, as every interval in $I^{\prime}(x)$ is disjoint from $*^{\prime}(x)$. Hence, $i \in 1^{\prime}(z)$ as well, and $p r_{z}^{\prime}(i)=p r_{x}^{\prime}(i)$. Also, let $\left[a_{1}, b_{1}\right]_{n}, \ldots,\left[a_{s}, b_{s}\right]_{n}$ be the maximal intervals in $I^{\prime}(x)$ contained in $\left[i_{u-1}, i_{u}\right]_{n}$. Then,

$$
c_{z}^{\prime}\left(i_{u-1}, i_{u}\right)=\sum_{l=1}^{s} c_{z}^{\prime}\left(a_{l}, b_{l}\right)=\sum_{l=1}^{s} c_{x}^{\prime}\left(a_{l}, b_{l}\right)=0 .
$$

Hence, $i_{u-1} \in 1^{\prime}(z), i_{u}=0^{\prime}(z)$ and $p r_{z}^{\prime}\left(i_{u-1}\right)=i_{u}$ as every element in $\left[i_{u-1}+1, i_{u}-1\right]_{n}$ has a pair in $x$. As $\left|*^{\prime}(z)\right|=2 k-n-2$, we must have $*^{\prime}(z)=*^{\prime}(x) \backslash\left\{i_{u-1}, i_{u}\right\}$ and so $I^{\prime}(z)=I^{\prime}(x) \cup\left\{\left[i_{u-1}, i_{u}\right]_{n}\right.$. The interval $\left[i_{u-1}, i_{u}\right]_{n}$ is also maximal, as no other interval of $I^{\prime}(z)$ contains $i_{u}$.

(ii) By the second part of (i), if $y \cup\{k\}>y$ for some $k \in[n]$, then

$$
k \in\left\{p r_{y}^{\prime}\left(j_{1}\right), \ldots, p r_{y}^{\prime}\left(j_{r}\right)\right\} .
$$

Let $k_{r}=p r_{y}^{\prime}\left(j_{r}\right)$ and $w=y \cup\left\{k_{r}\right\}$. Then, we have

$$
c_{w}^{\prime}(i, j)= \begin{cases}c_{y}^{\prime}(i, j) & \text { if } k_{r} \notin[i, j]_{n} \\ c_{y}^{\prime}(i, j)+2 & \text { if } k_{r} \in[i, j]_{n} .\end{cases}
$$

Let $a \in 1^{\prime}(y) \backslash\left\{j_{r}\right\}$. As $\left[j_{r}, k_{r}\right]$ is a maximal interval in $I^{\prime}(y)$, we have $k_{r} \notin\left[a, p r_{y}^{\prime}(a)\right]_{n}$. Hence, $c_{w}^{\prime}(a, b)=c_{y}^{\prime}(a, b)$ for all $b \in\left[a, p r_{y}^{\prime}(a)\right]_{n}$. But this means that $a \in 1^{\prime}(w)$ and $p r_{w}^{\prime}(a)=p r_{y}^{\prime}(a)$. Similarly, if $b \in 0^{\prime}(y) \backslash\left\{k_{r}\right\}$, then $b \in 0^{\prime}(w)$ and $p r_{w}^{\prime}(b)=p r_{y}^{\prime}(b)$.

The sets $1^{\prime}(w)$ and $0^{\prime}(w)$ have exactly $n-k$ elements, so we must have $1^{\prime}(w)=$ $1^{\prime}(y) \backslash\left\{j_{r}\right\}, 0^{\prime}(w)=0^{\prime}(y) \backslash\left\{k_{r}\right\}$ and $*^{\prime}(w)=*^{\prime}(y) \cup\left\{j_{r}, k_{r}\right\}$. Hence, by the first part of (i), we have $y<w$.

We finished analyzing the poset $(Q,<)$. Before we start the proof of Theorem 6 , let us mention the following well known properties of normalized matching graphs and posets. The first of these properties is the simple fact that in order to show that a poset has the normalized matching property, it is enough to concentrate on consecutive levels.

Proposition 12. Let $(P,<)$ be a graded poset with levels $A_{0}, \ldots, A_{n}$. For $k=0, \ldots, n-1$, let $G_{k}=\left(A_{k}, A_{k+1}, E_{k}\right)$ be the bipartite graph, where $x \in A_{k}$ and $y \in A_{k+1}$ are joined by an edge if $x<y$. If $G_{k}$ is a normalized matching graph for $k=0, \ldots, n-1$, then $(P,<)$ is a normalized matching poset.

The second property we shall use is less trivial, but it can be found in various sources $[3,13]$. We state it without proof.

Proposition 13. Let $G=(A, B, E)$ be a bipartite graph. The graph $G$ is a normalized matching graph if and only if there exist positive real numbers $a$ and $b$, and a weight function $w: E \rightarrow \mathbb{R}^{+}$such that for any $v \in A$ we have

$$
\sum_{\substack{v \in e \\ e \in E}} w(e)=a
$$


and for any $u \in B$ we have

$$
\sum_{\substack{u \in e \\ e \in E}} w(e)=b
$$

Now we are ready to prove Theorem 6 , namely that $(Q,<)$ is a normalized matching poset.

Proof of Theorem 6. By Proposition 12, it is enough to show that the bipartite subgraph of the comparability graph of $(Q,<)$ induced on $[n]^{(k-1)} \cup[n]^{(k)}$ is a normalized matching graph for $k=\lceil n / 2\rceil+1, \ldots, n$.

Fix $k$ with $n / 2+1 \leqslant k \leqslant n$, and let $G=\left([n]^{(k-1)},[n]^{(k)}, E\right)$ be the bipartite graph, where $x \in[n]^{(k-1)}$ and $y \in[n]^{(k)}$ are joined by an edge if $x<y$. We show that with the choice $a=2 n-2 k+2$ and $b=2 k$, there is a weight function $w: E \rightarrow \mathbb{R}^{+}$satisfying the conditions of Proposition 13.

Define $w: E \rightarrow \mathbb{R}^{+}$as follows. Suppose that $x \in[n]^{(k-1)}$ and $y \in[n]^{(k)}$ such that $x<y$ and let the elements of $*^{\prime}(y)$ be $i_{1} \cdots<i_{2 k-n}$. Then $x=y \backslash\left\{i_{u}\right\}$ for some $1 \leqslant u \leqslant 2 k-n$. Also, $\left[i_{u-1}, i_{u}\right]_{n}$ is a maximal interval in $I^{\prime}(x)$, where the index $u-1$ is meant modulo $2 k-n$. Let

$$
w(\{x, y\})=\left|\left[i_{u-1}, i_{u}\right]_{n}\right| .
$$

We show that $w$ suffices. Fix $x \in[n]^{(k-1)}$ and let $\left[j_{1}, p r_{x}^{\prime}\left(j_{1}\right)\right]_{n}, \ldots,\left[j_{t}, p r_{x}^{\prime}\left(j_{t}\right)\right]_{n}$ be the maximal intervals in $I^{\prime}(x)$. The neighbours of $x$ in $[n]^{(k)}$ are

$$
x \cup\left\{p r_{x}^{\prime}\left(j_{1}\right)\right\}, \ldots, x \cup\left\{p r_{x}^{\prime}\left(j_{t}\right)\right\} .
$$

Hence, we have

$$
\sum_{\substack{x \in e \\ e \in E}} w(e)=\sum_{u=1}^{t}\left|\left[j_{u}, p r_{x}^{\prime}\left(j_{u}\right)\right]_{n}\right| .
$$

But the intervals $\left[j_{1}, p r_{x}^{\prime}\left(j_{1}\right)\right]_{n}, \ldots,\left[j_{t}, p r_{x}^{\prime}\left(j_{t}\right)\right]_{n}$ are pairwise disjoint and their union is $[n] \backslash *^{\prime}(x)$, so

$$
\sum_{u=1}^{t}\left|\left[j_{u}, p r_{x}^{\prime}\left(j_{u}\right)\right]_{n}\right|=\left|[n] \backslash *^{\prime}(x)\right|=2 n-2 k+2=a .
$$

Now fix $y \in[n]^{(k)}$ and let $i_{1} \cdots<i_{2 k-n}$ be the elements of $*^{\prime}(y)$. The neighbours of $y$ in $G$ are

$$
y \backslash\left\{i_{1}\right\}, \ldots, y \backslash\left\{i_{2 k-n}\right\} .
$$

Hence,

$$
\sum_{\substack{y \in e \\ e \in E}} w(e)=\sum_{u=1}^{2 k-n}\left|\left[i_{u-1}, i_{u}\right]_{n}\right|
$$


where the index $u-1$ is taken modulo $2 k-n$. Every element of $[n] \backslash *^{\prime}(x)$ is contained in exactly one of the intervals $\left[i_{1}, i_{2}\right]_{n}, \ldots,\left[i_{2 k-n}, i_{1}\right]_{n}$, while every element of $*^{\prime}(x)$ is contained in exactly two of those intervals. Hence,

$$
\sum_{u=1}^{2 k-n}\left|\left[i_{u-1}, i_{u}\right]_{n}\right|=2 k=b .
$$

So the weight function $w$ suffices and $(Q,<)$ is a normalized matching poset.

\section{Partitioning the Boolean lattice into rank-symmetric chains of uniform size}

In this section, we prove Theorem 4. In the proof, we apply the following two theorems from [16].

Theorem 14. Let $P$ be an unimodal normalized matching poset of width $w$. The poset $P$ can be partitioned into $w$ chains, each chain of size at most $\frac{2|P|}{w}+5$.

Theorem 15. Let $P$ be a normalized matching poset with levels $A_{0}, A_{1}, \ldots, A_{n}$ and let $a_{i}=\left|A_{i}\right|$ for $i=0, \ldots, n$. Suppose that $w=a_{0} \geqslant a_{1} \geqslant \ldots \geqslant a_{n}$. Let $f:\{0, \ldots, n\} \rightarrow$ $\mathbb{N} \cup\{\infty\}$ be defined by

$$
f(k)=\min \left\{i>k: a_{k+1}+\ldots+a_{i} \geqslant w\right\},
$$

where $a_{i}=0$ if $i>n$. Also, let $f_{1}=f$ and $f_{i}=f \circ f_{i-1}$ denote the iterations of $f$; set $f_{0} \equiv 0$. Finally, let $d$ be the largest integer such that $f_{d}(0)<\infty$. The poset $P$ can be partitioned into $w$ chains of size at least $d+1$.

The proof of Theorem 4 is almost the same as the proof of Theorem 1.5 in [16]. For completeness, we provide the proof here as well, but we shall copy most of it word by word.

Proof of Theorem 4. Again, let $Q=[n]^{(\geqslant n / 2)}$ and choose a bijection $p: Q \rightarrow[n]^{(\leqslant n / 2)}$ and partial ordering $<$ such that they satisfy the conditions of Theorem 1 . The width of $(Q,<)$ is $w=\left(\begin{array}{c}n \\ \lfloor n / 2\rfloor\end{array}\right)$ as the symmetric chain decomposition of $2^{[n]}$ also defines a chain decomposition of $(Q,<)$ into $w$ chains. (It also follows from the normalized matching property.) For $i=0, \ldots,\lfloor n / 2\rfloor$, let $A_{i}=[n]^{(\lceil n / 2\rceil+i)}$. First, we need some approximation of the size of the level $A_{i}$, where $i=O(\sqrt{n})$.

Using Stirling's approximation one can easily get the estimation that for any $t \in \mathbb{R}$ we have

$$
\left(\begin{array}{c}
n \\
n / 2+t \sqrt{n}
\end{array}\right)=(1+o(1)) 2^{n} e^{-2 t^{2}} \sqrt{\frac{2}{\pi n}}=(1+o(1)) e^{-2 t^{2}} w
$$

as $n \rightarrow \infty$. 
For $k=1,2, \ldots$, let $T_{k}>0$ be the smallest integer such that $\left(\begin{array}{c}n \\ \lceil n / 2\rceil+T_{k}\end{array}\right)<w / k$. Then, by (1) we have $T_{k}=(1 / \sqrt{2}+o(1)) \sqrt{n} \sqrt{\log k}$.

Let $K$ be the smallest positive integer such that

$$
\alpha-\frac{\epsilon}{2}<\sqrt{2} \sum_{k=2}^{K} \frac{\sqrt{\log k}-\sqrt{\log (k-1)}}{k} .
$$

Note that for $k>2$ we have

$$
\frac{\sqrt{\log k}-\sqrt{\log (k-1)}}{k}=\frac{(\log k)-(\log (k-1))}{k(\sqrt{\log (k-1)}+\sqrt{\log k})}<\frac{1}{k^{2}} .
$$

Hence, the tail of the sum $\sum_{k=2}^{\infty} \frac{\sqrt{\log k}-\sqrt{\log (k-1)}}{k}$ can be easily bounded:

$$
\sum_{k=K+1}^{\infty} \frac{\sqrt{\log k}-\sqrt{\log (k-1)}}{k}<\sum_{k=K+1}^{\infty} \frac{1}{k^{2}}<\frac{1}{K}
$$

This implies $K=O(1 / \epsilon)$.

Let $P$ be the subposet of $(Q,<)$ induced by the levels $A_{0}, \ldots, A_{T_{K}}$. Define $f$ and $d$ as in Theorem 15. For $k=2,3, \ldots, K$, if $T_{k-1}<j \leqslant T_{k}-k$, then $f(j)=j+k$. Hence,

$$
\begin{aligned}
d & \geqslant \sum_{k=2}^{K}\left\lfloor\frac{T_{k}-T_{k-1}-k}{k}\right\rfloor=\left(\frac{1}{\sqrt{2}}+o(1)\right) \sqrt{n} \sum_{k=2}^{K} \frac{\sqrt{\log k}-\sqrt{\log (k-1)}}{k} \\
& \geqslant\left(\frac{1}{2}+o(1)\right) \sqrt{n}\left(\alpha-\frac{\epsilon}{2}\right) .
\end{aligned}
$$

Thus, by Theorem 15 there exists a chain partition of $P$ into $w$ chains, each of size at least $(1 / 2+o(1)) \sqrt{n}(\alpha-\epsilon / 2)$ and at most

$$
T_{K}=\left(\frac{1}{\sqrt{2}}+o(1)\right) \sqrt{n} \sqrt{\log K} .
$$

Let $\left\{C_{x}\right\}_{x \in B_{\lceil n / 2\rceil}}$ be such a partition with $x \in C_{x}$ for all $x \in B_{\lceil n / 2\rceil}$.

Let $P^{\prime}$ be the subposet of $Q$ induced by the levels $A_{T_{K}}, \ldots, A_{\lfloor n / 2\rfloor}$. Then $P^{\prime}$ is also a unimodal normalized matching poset with width

$$
w^{\prime}=\left|A_{T_{K}}\right|=\left(\frac{1}{K}+o(1)\right) w .
$$

Thus, by Theorem 14, $P^{\prime}$ has a partition into $w^{\prime}$ chains, each of size at most

$$
\frac{2\left|P^{\prime}\right|}{w^{\prime}}+5=\frac{2(K+o(1))|Q|}{w}<\frac{(K+o(1)) 2^{n}}{w}=(K+o(1)) \sqrt{\frac{\pi n}{2}} .
$$


Let $\left\{D_{y}\right\}_{y \in B_{\lceil n / 2\rceil+T_{K}}}$ be such a partition with $y \in D_{y}$ for all $y \in B_{\lceil n / 2\rceil+T_{K}}$.

For all $x \in B_{\lceil n / 2\rceil}$, let $C_{x}^{\prime}=C_{x}$ if $C_{x} \cap A_{T_{K}}=\emptyset$ and let $C_{x}^{\prime}=C_{x} \cup D_{y}$ if $C_{x} \cap A_{T_{K}}=\{y\}$. Then $\left\{C_{x}^{\prime}: x \in A_{0}\right\}$ is a partition of $Q$ satisfying

$$
\left(\frac{1}{2}+o(1)\right)\left(\alpha-\frac{\epsilon}{2}\right) \sqrt{n}<\left|C_{x}\right|<T_{K}+(K+o(1)) \sqrt{\frac{\pi n}{2}} .
$$

for all $x \in A_{0}$. Here, the right hand side equals to

$$
\left(\sqrt{\frac{\log K}{2}}+K \sqrt{\frac{\pi}{2}}+o(1)\right) \sqrt{n}=O\left(\frac{\sqrt{n}}{\epsilon}\right) .
$$

Hence, $\left\{C_{x}^{\prime} \cup p\left(C_{x}^{\prime}\right): x \in A_{0}\right\}$ is a chain partition of $2^{[n]}$ into rank-symmetric chains of size between $(\alpha-\epsilon / 2+o(1)) \sqrt{n}$ and $O(\sqrt{n} / \epsilon)$.

Setting $\epsilon=0.04$, one can get the exact bounds $0.8 \sqrt{n}$ and $13 \sqrt{n}$ for sufficiently large $n$. We shall avoid doing these calculations.

\section{The number of symmetric chain partitions of the Boolean lattice}

In this section, we present bounds on the number of symmetric chain decompositions of $2^{[n]}$ and we prove Theorem 5 . First, we show a short proof of a slightly worse bound than the one we have in Theorem 5, without using Theorem 1. This simple proof exploits the inductive method we used to define the CSCD.

Proposition 16. The Boolean lattice $2^{[n]}$ has at least

$$
2^{\Omega\left(2^{n} / \sqrt{n}\right)}
$$

symmetric chain decompositions.

Proof. Let $m=\left(\begin{array}{c}n-1 \\ \lfloor(n-1) / 2\rfloor\end{array}\right)$ and let $C_{1}, \ldots, C_{m}$ be a symmetric chain decomposition of $2^{[n-1]}$ such that $\left|C_{1}\right| \geqslant \cdots \geqslant\left|C_{m}\right|$. Let $1 \leqslant l \leqslant m$ be the largest integer such that $\left|C_{l}\right|>1$, then

$$
l=\left(\begin{array}{c}
n-1 \\
\lfloor(n-2) / 2\rfloor
\end{array}\right)=\Theta\left(2^{n} / \sqrt{n}\right) .
$$

For each sequence $\left(i_{1}, \ldots, i_{l}\right) \in\{0,1\}^{l}$, we define the following symmetric chain partition of $2^{[n]}$.

For $j=l+1, \ldots, m$, let $C_{j}^{1}=\{x, x \cup\{n\}\}$, where $x$ is the only element of $C_{j}$. For $j \in[l]$, let the elements of $C_{j}$ be $x_{1} \subset \cdots \subset x_{k}$. If $i_{j}=0$, let

$$
\begin{gathered}
C_{j}^{1}=\left\{x_{1}, \ldots, x_{k}, x_{k} \cup\{n\}\right\}, \\
C_{j}^{2}=\left\{x_{1} \cup\{n\}, \ldots, x_{k-1} \cup\{n\}\right\} ;
\end{gathered}
$$


and if $i_{j}=1$, let

$$
\begin{gathered}
C_{j}^{1}=\left\{x_{1}, x_{1} \cup\{n\}, \ldots, x_{k} \cup\{n\}\right\}, \\
C_{j}^{2}=\left\{x_{2}, \ldots, x_{k}\right\} .
\end{gathered}
$$

Then one can easily check that $C_{1}^{1}, \ldots, C_{m}^{1}, C_{1}^{2}, \ldots C_{l}^{2}$ is a symmetric chain decomposition of $2^{[n]}$, and for each sequence $\left(i_{1}, \ldots, i_{l}\right) \in\{0,1\}^{l}$ we get a different partition. Hence, we showed that $2^{[n]}$ has at least $2^{l}=2^{\Omega\left(2^{n} / \sqrt{n}\right)}$ symmetric chain decompositions.

Now we show that we can gain an extra $\log n$ factor in the exponent by utilizing Theorem 1.

Let $Q=[n]^{(\geqslant n / 2)}$, and choose a bijection $p$ and partial ordering $<$ satisfying the conditions in Theorem 1. Also, for simplicity, write $M=\lceil n / 2\rceil$ and $w=\left(\begin{array}{c}n \\ M\end{array}\right)$. If $C \subset Q$ is a skipless chain with minimal element lying in $[n]^{M}$, then $C \cup p(C)$ is a symmetric chain in $2^{[n]}$.

For $i=M, \ldots, n-1$, let $G_{i}$ be the comparability graph of the subposet of $(Q,<)$ induced on the levels $[n]^{(i)}$ and $[n]^{(i+1)}$. Then $G_{i}$ is a normalized matching graph. Let $\mathfrak{T}_{i}$ be the set of complete matchings from $[n]^{(i+1)}$ to $[n]^{(i)}$ in $G_{i}$. Note that each sequence of matchings $\left(T_{M}, \ldots, T_{n-1}\right) \in \mathfrak{T}_{M} \times \cdots \times \mathfrak{T}_{n-1}$ corresponds to a unique partition of $Q$ into skipless chains with minimal elements in $[n]^{(M)}$. Look at the graph with vertex set $Q$, where two elements are joined by an edge if they are joined by an edge in one of $T_{M}, \ldots, T_{n-1}$. Then the components of this graph are skipless chains, and every component intersects $[n]^{(M)}$. Hence, the components of this graph form a chain partition of $Q$ into skipless chains with minimal elements in $[n]^{(M)}$.

Thus, every element of $\mathfrak{T}_{M} \times \cdots \times \mathfrak{T}_{n-1}$ corresponds to a unique symmetric chain partition of $2^{[n]}$. Our task is reduced to estimating the size of $\mathfrak{T}_{i}$ for $i=M, \ldots, n-1$.

Theorem 17. Let $G=(A, B, E)$ be a normalized matching graph with $|A| \leqslant|B|$. The number of complete matchings from $A$ to $B$ is at least $\sqrt{\left(\begin{array}{l}|B| \\ |A|\end{array} \mid\right.}$.

Proof. Let $|A|=k$ and $|B|=n$. Let

$$
M(G)=\left\{V \in B^{(k)}: \exists \text { complete matching from } A \text { to } V \text { in } G\right\} .
$$

It is enough to show that $M(G) \geqslant \sqrt{\left(\begin{array}{l}n \\ k\end{array}\right)}$.

We use the following observation: let $G^{\prime}=\left(A, B, E^{\prime}\right)$ be another normalized matching graph on the same vertex set. Then $M(G) \cap M\left(G^{\prime}\right) \neq \emptyset$. To prove this observation, we use the following well known result of Griggs [7]: every rank-symmetric, unimodal normalized matching poset has a symmetric chain partition.

Create the following graded poset $(P,<)$ : let the levels of $P$ be $A, B, A^{\prime}$, where $A^{\prime}$ is a disjoint copy of $A$; let the partial order $<$ be induced by the following relations: for $a \in A$, $a^{\prime} \in A^{\prime}$ and $b \in B$ let $a<b$ if $a b \in E$, and $b<a^{\prime}$ if $a^{\prime} b \in E^{\prime}$. Then $(P,<)$ is a unimodal, rank-symmetric normalized matching poset, so it has a symmetric chain decomposition. This chain decomposition contains chains of size 1 and 3. Let $V \subset B$ be the set of elements which are the middle elements of chains of size 3. Then $V \in M(G) \cap M\left(G^{\prime}\right)$. 
Let $S_{B}$ denote the set of all permutations of $B$. For $\pi \in S_{B}$ define the bipartite graph $G_{\pi}=\left(A, B, E_{\pi}\right)$ such that $a \in A$ and $b \in B$ are joined by an edge if $a \pi(b) \in E$.

Clearly, $G_{\pi}$ is a normalized matching graph as $G_{\pi}$ is isomorphic to $G$. Hence, we have that $M(G) \cap M\left(G_{\pi}\right) \neq \emptyset$. Note that

$$
M\left(G_{\pi}\right)=\left\{\pi^{-1}(V): V \in M(G)\right\},
$$

so for each $\pi \in S_{B}$ there exists a pair $(V, W) \in M(G) \times M(G)$ such that $V=\pi^{-1}(W)$. But for each pair $(V, W)$ there are exactly $k !(n-k)$ ! permutations $\pi \in S_{B}$ such that $V=\pi^{-1}(W)$. So, we must have

$$
k !(n-k) !|M(G)|^{2} \geqslant n !
$$

Remark. The result of Theorem 17 is not sharp for any $1 \leqslant k \leqslant n-1$. However, we cannot replace $\sqrt{\left(\begin{array}{l}n \\ k\end{array}\right)}$ in the theorem with any number larger than $\left(\begin{array}{l}n \\ k\end{array}\right)$. This is true as every minimal normalized matching graph is a forest (see [3]), and in this case the number of complete matchings from $A$ to $B$ is equal to $|M(G)|$. Note that $|M(G)| \leqslant\left(\begin{array}{l}n \\ k\end{array}\right)$ trivially holds.

Proof of Theorem 5. Write $a_{k}$ for $\left(\begin{array}{l}n \\ k\end{array}\right)$. We have already established that $2^{[n]}$ has at least $\left|\mathfrak{T}_{M}\right| \ldots\left|\mathfrak{T}_{n-1}\right|$ symmetric chain decompositions. Also, by Theorem 17, we have $\left|\mathfrak{T}_{k}\right| \geqslant$ $\sqrt{\left(\begin{array}{c}a_{k} \\ a_{k+1}\end{array}\right)}$ for $k=M, \ldots, n-1$. We shall estimate $\left(\begin{array}{c}a_{k} \\ a_{k-1}\end{array}\right)$ for $n / 2+\sqrt{n}<k<n / 2+2 \sqrt{n}$. In this case, we have $a_{k}=\Theta\left(2^{n} / \sqrt{n}\right)$ and $a_{k}-a_{k+1}=\Theta\left(2^{n} / n\right)$. Hence, using the well known lower bound $\left(\begin{array}{l}a \\ b\end{array}\right) \geqslant(a / b)^{b}$ for binomial coefficients, we get

$$
\begin{aligned}
\left(\begin{array}{c}
a_{k} \\
a_{k+1}
\end{array}\right) & =\left(\begin{array}{c}
a_{k} \\
a_{k}-a_{k+1}
\end{array}\right) \geqslant\left(\frac{a_{k}}{a_{k}-a_{k+1}}\right)^{a_{k}-a_{k-1}} \\
& =\sqrt{n}^{\Theta\left(2^{n} / n\right)}=2^{\Theta\left(2^{n} \log n / n\right)} .
\end{aligned}
$$

Thus, we have

$$
\prod_{k=M}^{n-1}\left|\mathfrak{T}_{k}\right| \geqslant \prod_{n / 2+\sqrt{n}<k<n / 2+2 \sqrt{n}} \sqrt{\left(\begin{array}{c}
a_{k} \\
a_{k+1}
\end{array}\right)}=2^{\Omega\left(2^{n} \log n / \sqrt{n}\right)}
$$

We note that $2^{[n]}$ has at most $2^{O\left(2^{n} \log n\right)}$ symmetric chain decompositions. Furthermore, it has at most $2^{O\left(2^{n} \log n\right)}$ chain decompositions into $\left(\begin{array}{c}n \\ \lfloor n / 2\rfloor\end{array}\right)$ skipless chains. We can get the upper bound $n^{2^{n}}$ by the following simple observation: every chain partition of $2^{[n]}$ into $\left(\begin{array}{c}n \\ \lfloor n / 2\rfloor\end{array}\right)$ skipless chains corresponds to a unique sequence $\left(U_{0}, \ldots, U_{n-1}\right)$, where $U_{k}$ is a complete matching from $[n]^{(k)}$ to $[n]^{(k+1)}$ for $k<M$, and $U_{k}$ is a complete matching from 
$U_{k+1}$ to $U_{k}$ for $k \geqslant M$. But for each $k$, the number of such matchings is less than $n\left(\begin{array}{l}n \\ k\end{array}\right)$, as the comparability subgraph of $2^{[n]}$ induced on the levels $[n]^{(k)} \cup[n]^{(k+1)}$ has maximum degree at most $n$. Hence, the number of symmetric chain decompositions of $2^{[n]}$ is less than $\prod_{k=0}^{n} n^{\left(\begin{array}{l}n \\ k\end{array}\right)}=n^{2^{n}}$.

Let us mention that an unpublished result of Balogh and Wagner shows that the number of chain decompositions of $2^{[n]}$ into $\left(\begin{array}{c}n \\ \lfloor n / 2\rfloor\end{array}\right)$ skipless chains is exactly $2^{\Theta\left(2^{n} \log n\right)}$.

\section{Open problems}

In this section, we propose some open problems.

After the method we used to prove Theorem 4, it is natural to ask the following question. Let $P$ be a rank-symmetric, unimodal normalized matching poset of width $w$. Can we partition $P$ into $w$ rank-symmetric chains such that the sizes of the chains are as close to each other as possible?

As we do not know the answer in the simpler case when our chains are not necessarily rank-symmetric, we ask a less ambitious question.

Question 18. Define the function $f: \mathbb{Q}^{+} \rightarrow \mathbb{N}$ as follows: for $r \in \mathbb{Q}^{+}, f(r)$ is the maximal positive integer such that any rank-symmetric, unimodal normalized matching poset $P$ of width $|P| / r$ can be partitioned into rank-symmetric chains of size at least $f(r)$. Is it true that $\lim _{r \rightarrow \infty} f(r)=\infty$ ?

Another closely related question is motivated by the following theorem of Lonc [14]. He proved that if given a positive integer $h$ and $n$ is sufficiently large, then $2^{[n]}$ has a chain partition, where all but at most one of the chains have size $h$. The author of this paper [17] showed that the smallest such $n$ is $O\left(h^{2}\right)$ and this bound is best possible up to a constant. We propose the following rank-symmetric version of this problem.

Conjecture 19. For every positive integer $h$ there exists a positive integer $N(h)$ such that if $n>N(h)$ and $n$ is odd, then $2^{[n]}$ has a partition into rank-symmetric chains, where all but at most one of the chains have size $2 h$.

We note the following connection between the results of this paper and Conjecture 19. Suppose that the following conjecture is true.

Conjecture 20. There exists some real $t>0$ such that taking $k=\lfloor n / 2+t \sqrt{n}\rfloor$, the bipartite subgraph of $(Q,<)$ induced on $[n]^{(k)}$ and $[n]^{(k+1)}$ has a spanning tree with maximum degree $o(\sqrt{n})$.

Then Conjecture 19 follows from Theorem 4. Surprisingly, we have not been able to prove the much weaker statement that the bipartite subgraph of $(Q,<)$ induced on $[n]^{(k)}$ and $[n]^{(k+1)}$ is connected for $k=n / 2+\Theta(\sqrt{n})$. We note that if $n$ is even, then the bipartite subgraph of $(Q,<)$ induced on the levels $[n]^{(n / 2)}$ and $[n]^{(n / 2+1)}$ is not even connected, it is the union of trees of size $n+1$. 


\section{Acknowledgements}

I would like to thank the referee for the useful comments and suggestions.

\section{References}

[1] I. Anderson. Combinatorics of Finite Sets. Oxford University Press, 1987.

[2] N. G. De Brujin, C. van Ebbenhorst Tengbergen, D. Kruyswijk. On the set of divisors of a number. Nieuw Arch. Wiskunde, 23(2):191-193, 1951.

[3] D. E. Daykin, L. H. Harper, D. B. West. Some Remarks on Normalized Matching. Journal of Combinatorial Theory A, 35:301-308, 1983.

[4] R. P. Dilworth. A Decomposition Theorem for Partially Ordered Sets. Annals of Mathematics, 51(1):161-166, 1950.

[5] Z. Füredi. Problem session. Kombinatorik geordneter Mengen, Oberwolfach, B.R.D., 1985.

[6] C. Greene, D. J. Kleitman. Strong versions of Sperner's theorem. Journal of Combinatorial Theory A, 20(1):80-88, 1976.

[7] J. R. Griggs. Sufficient conditions for a symmetric chain order. SIAM J. Appl. Math., 32(4):807-809, 1977.

[8] P. Hall. On Representatives of Subsets. J. London Math. Soc., 10(1):26-30, 1935.

[9] T. Hsu, M. J. Logan, S. Shahriari, C. Towse. Partitioning the Boolean lattice into chains of large minimum size. Journal of Combinatorial Theory A, 97(1):62-84, 2002.

[10] T. Hsu, M. J. Logan, S. Shahriari, C. Towse. Partitioning the Boolean lattice into a minimal number of chains of relatively uniform size. European Journal of Combinatorics, 24:219-228, 2003.

[11] K. K. Jordan. The necklace poset is a symmetric chain order. Journal of Combinatorial Theory A, 117:625-641, 2010.

[12] D. J. Kleitman. On a lemma of Littlewood and Offord on the distribution of certain sums. Mathematische Zeitschrift, 90(4):251-259, 1965.

[13] D. J. Kleitman. On An Extremal Property of Antichains in Partial Orders. The Lym Property and Some of Its Implications and Applications. NATO Advanced Study Institutes Series, 16:277-290, 1975.

[14] Z. Lonc. Proof of a Conjecture on Partitions of a Boolean Lattice. Order, 8:11-21, 1991.

[15] E. Sperner. Ein Satz über Untermengen einer endlichen Menge. Mathematische Zeitschrift (in German), 27(1):544-548, 1928.

[16] I. Tomon. On a conjecture of Füredi. European Journal of Combinatorics, 49:1-12, 2015.

[17] I. Tomon. Improved bounds on the partitioning of the Boolean lattice into chains of equal size. Discrete Mathematics, 339(1):333-343, 2016. 